\title{
Erratum to: Association of cortical shape of the mandible on panoramic radiographs with mandibular trabecular bone structure in Japanese adults: a cone-beam CT-image analysis
}

\author{
Noriyasu Mochizuki $\cdot$ Noriyuki Sugino $\cdot$ \\ Tadashi Ninomiya $\cdot$ Nobuo Yoshinari $\cdot$ \\ Nobuyuki Udagawa $\cdot$ Akira Taguchi
}

Published online: 11 December 2013

(C) Japanese Society for Oral and Maxillofacial Radiology and Springer Japan 2013

\section{Erratum to: Oral Radiol \\ DOI 10.1007/s11282-013-0155-z}

Unfortunately, the above mentioned article was published incorrectly with errors in its original publication. It is corrected as follows,

1. In the abstract section, last sentence of results should read "However, trabecular bone volume fraction increased significantly with age $(P=0.017)$."

2. Table 2 and Fig. 6 were erroneously published, the correct Table 2 and Fig. 6 are in the next page.

The online version of the original article can be found under doi:10.1007/s11282-013-0155-z.

N. Mochizuki · A. Taguchi

Department of Hard Tissue Research, Graduate School of Oral

Medicine, Matsumoto Dental University,

Shiojiri 399-0781, Japan

N. Mochizuki $\cdot$ N. Sugino $\cdot$ A. Taguchi $(\bowtie)$

Department of Oral and Maxillofacial Radiology, School of Dentistry, Matsumoto Dental University, 1780 Gobara, Hirooka,

Shiojiri 399-0781, Japan

e-mail: akiro@po.mdu.ac.jp

T. Ninomiya $\cdot$ N. Udagawa

Institute for Oral Science, Matsumoto Dental University,

Shiojiri 399-0781, Japan

\section{N. Yoshinari}

Department of Periodontology, Matsumoto Dental University, Shiojiri 399-0781, Japan

N. Udagawa

Department of Biochemistry, Matsumoto Dental University,

Shiojiri 399-0781, Japan
3. In the results section,

(a) Third sentence of the 2nd paragraph should read,

Conversely, subjects with a severely eroded cortex had a significantly higher trabecular bone volume fraction than those with a normal $(P<0.001)$ or mildly to moderately eroded cortex $(P=0.001)$; however, no significant difference was observed between subjects with a normal cortex and those with a mildly to moderately eroded one (Fig. 4).

(b) The third paragraph should read,

The cortical bone volume fraction decreased significantly with age $(P=0.04$; Fig. 5$)$, while the trabecular bone volume fraction of the mandible increased significantly with age $(P=0.017$; Fig. 6$)$. There were no significant correlations between age and trabecular bone volume fraction in the 22 subjects with a normal cortex $(r=0.279, P=0.208)$, in the 18 subjects with a mildly to moderately eroded cortex $(r=0.226, P=0.367)$, and in the ten subjects with a severely eroded cortex $(r=-0.373$, $P=0.288)$. There was no significant correlation in the 18 men $(r=0.360, P=0.142)$, but age tended to be correlated with trabecular bone volume fraction in the 32 women $(r=0.314, P=0.080)$.

4. In the discussion section,

(a) The first paragraph, from "The cortical bone volume fraction" to "younger women with a normal cortex." should be,

The cortical bone volume fraction of the mandible decreased significantly with age, although the trabecular bone volume fraction increased significantly with age. We determined that the correlation between age and trabecular bone volume fraction in women may contribute to this phenomenon. Older women may have a higher trabecular bone volume fraction than younger women. 
Table 2 Differences in total bone volume (TV), cortical bone volume fraction (CVF), trabecular bone volume fraction (TVF), and fractal dimension (FD) among the three cortical shape groups, as determined on panoramic radiographs

\begin{tabular}{|c|c|c|c|c|}
\hline & Normal & Mildly to moderately eroded & Severely eroded & $P$ \\
\hline $\mathrm{TV}\left(\mathrm{mm}^{3}\right)$ & $2674.5 \pm 71.1$ & $2566.5 \pm 103.2$ & $2525.5 \pm 133.5$ & 0.53 \\
\hline $\operatorname{CVF}(\%)$ & $57.0 \pm 1.8^{\mathrm{a}}$ & $56.4 \pm 1.7^{\mathrm{b}}$ & $37.0 \pm 2.8^{\mathrm{a}, \mathrm{b}}$ & $<0.001$ \\
\hline $\operatorname{TVF}(\%)$ & $15.9 \pm 6.8^{\mathrm{a}}$ & $18.5 \pm 5.6^{\mathrm{c}}$ & $28.7 \pm 8.7^{\mathrm{a}, \mathrm{c}}$ & $<0.001$ \\
\hline FD & $2.31 \pm 0.02^{\mathrm{d}}$ & $2.38 \pm 0.02$ & $2.40 \pm 0.02^{\mathrm{d}}$ & 0.010 \\
\hline
\end{tabular}

Results are shown as mean \pm standard error of mean

a,b $P<0.001,{ }^{\mathrm{c}} P=0.001,{ }^{\mathrm{d}} P<0.05$

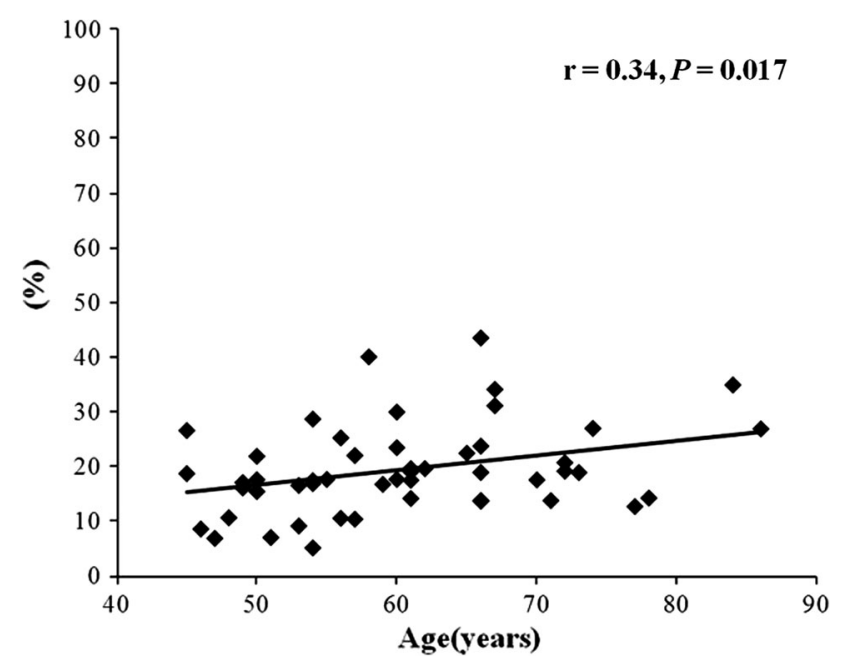

Fig. 6 Correlation between age and trabecular bone volume fraction (b) The 2nd sentence of the last paragraph should read, The cortical bone volume fraction decreased significantly with age, but the trabecular bone volume fraction increased significantly with age. 